\title{
Injection techniques in SFC
}

\author{
T. Greibrokk ${ }^{1}$ and T.L. Chester ${ }^{2}$ \\ ${ }^{1}$ Department of Chemistry, University of Oslo, POB 1033 Blindern, 0315 Oslo, Norway \\ ${ }^{2}$ The Procter \& Gamble Company, POB 538707, Cincinnati, Ohio 45253-8707, USA
}

In SFC, as well as in any form of chromatography, the key to understanding injection rests in understanding the mass transfer processes. This includes the formation of mixtures with the mobile phase and the phase transitions the sample may experience at the column inlet. Critical loci for type-I binary mixtures with $\mathrm{CO}_{2}$ are reported, enabling direct injection on a retention gap. With dilute sample solutions, peak focusing at the column inlet is required, either by phase focusing, phase ratio focusing or solvent trapping, using retention gaps on precolumns connected to the column. Advantages and disadvantages of different injection techniques have been described, with particular reference to techniques for open tubular columns. Injection volumes on regular size packed HPLC columns are less critical, compared to open tubular columns, but the basic parameters concerning phase behaviour and solvent strength are valid for packed columns as well.

Keywords: SFC, injection, sample, introduction

\section{Introduction}

The objective of the injector is to transfer the whole sample or a predetermined fraction of the sample quantitatively to the column in the narrowest possible band. The first part of this objective, a quantitative transfer of all components, is not always obtained since sample discrimination occurs in some injection techniques in SFC as well as in GC. The second part of the objective, transfer in a narrow band, is a process which is affected by numerous factors, such as diffusion, linear velocity, temperature, solvents, loading and mixing efficiency, as well as phase transitions. Some of these factors also influence the first part of the objective, the yield of the transfer process. Injector temperatures exceeding room temperature are only used with solutes which need higher temperature to stay in solution, such as some polymers. Injection at high temperature should be performed with caution, since a solvent which starts to evaporate from a hot injector, before the valve is actuated, may result in sample loss and poor reproducibility.

In most applications the sample is introduced as a relatively large (depending on the analyte concentration) liquid plug into the stream of mobile phase. Due to limited mixing, the major part of the sample often arrives at the column inlet dissolved in a liquid composed of the solvent partially mixed with the mobile phase. Thus, the transient elution strength of the sample may often be considerably higher than the elution strength of the mobile phase, and can adversely affect the peak shapes by flooding and dispersing the sample over the column inlet.

It is well known that an exponential decay injection profile is obtained because of the laminar flow of the displacing mobile phase [1]. By moving the valve back to the load position from the inject position after a predetermined time, the solvent tail can be removed. The injection syringe should be removed first, since the pressure in the sample loop is released through the injection and waste ports when the valve is returned to the load position. With large sample loops the syringe might be pushed out of the port, or the plunger pushed out of the barrel, if the syringe is left in the injection port.

By injecting very small volumes, using splitting or small sample loops, a more thorough mixing with the mobile phase can be achieved prior to the column. Currently the nominal volume of the smallest commercially available sample loop is $60 \mathrm{nl}$. However, small volume $(<100 \mathrm{nl})$ internal sample loops are difficult to manufacture with high accuracy, and the small volumes are difficult to maintain without changes, particularly with loops made from polymeric materials.

Column overload is rarely caused by the mass of the solutes, but almost always by the amount of solvent. With a retention factor $\mathrm{k}=1$, the injected volume that produces $10 \%$ peak broadening has been calculated to be $24 \mathrm{nl}$ on a $50-\mu \mathrm{m}-\mathrm{ID}$ open-tubular column, and with a retention factor of 10, the volume was calculated to be $130 \mathrm{nl}$ [2]. With an actual injection of $223 \mathrm{nl}, 10 \%$ loss of resolution was measured for a solute with $\mathrm{k}=3.2$ on a $50-\mu \mathrm{m}-\mathrm{ID}$ column [3].

The main disadvantage of being forced to introduce small volumes is the requirement for concentrated sample solutions. This removes the possibility to do trace analysis. The highly concentrated solutions also increase the memory effects in subsequent injections. Solute concentrations down to $5 \mathrm{ppm}$ were analyzed with a standard system with a $200 \mathrm{nl}$ loop, direct injection and a detector with a detection limit of $0.1 \mathrm{ng}$. With split injection the limit was determined to $150 \mathrm{ppm}$ [4]. At lower concentrations, solvent removal is required, either by solvent venting or by evaporation from a flooded retention gap. 


\section{Aspects of phase behavior pertaining to injection processes}

In doing chromatography we seldom deal with pure fluids. Mobile phases are often binary or even ternary mixtures. Even when the mobile phase is composed entirely of a single chemical, we at least temporarily form mixtures when injecting samples. The key to understanding injection in SFC, or in any form of chromatography, rests in understanding the mass transfer processes. This understanding must include the phase transitions the sample may experience during its trip to the column inlet.

Our goal is to simplify that general description, considering only the cases most often encountered in analytical SFC. Injection is most difficult in open-tubular SFC because of the extremely small dimensions and the relative ease of flooding the column with even sub-microliter sample injections if a mistake is made. However, injection is extremely simple and reliable as long as initial parameters are set within the limits imposed by the phase behavior of the mobile phase-sample solvent mixture. As we shall see, this is very easy to do.

The simplest binary mixtures are Type I mixtures [5] in which the two separate components are miscible as liquids. For these mixtures the critical points of the two pure mixture components are joined in a three-dimensional pressuretemperature-composition phase diagram by a continuous locus of mixture critical points, as shown in figure 1. A twophase liquid-vapor region exists as a solid volume in this three-dimensional space. However, when we use a singlecomponent mobile phase in SFC and inject into it a different sample solvent, all the phase behavior information we need is available by considering only a projection of the three-dimensional phase diagram for the resulting mixture. The projection is made along the composition axis, and results in a pressure-temperature plot, as shown in figure 2 . Here we see the boiling curves for the two pure components of interest (that is, the pure mobile phase and the sample solvent) ending at their critical points, and the projection of the locus of the mixture critical points. The significance of this plot to us is that all points outside the critical locus constitute a region of temperature and pressure where a liquidvapor phase separation can never occur for this mixture, regardless of how the concentration ratio of the two components might change. However, for points inside the critical locus, liquid-vapor phase separation is possible, depending on the mixture composition. When neat sample solvent (or a dilute sample) is injected as a plug into a stream of mobile phase, at points not too far from the injector the composition will span the complete composition range as the plug passes, thereby assuring us that a phase separation will occur if the temperature and pressure at that point are within the bounds of the critical locus.

We will limit our discussion to Type I mixtures because $\mathrm{CO}_{2}$, the most frequently used mobile phase in SFC, forms Type I mixtures with many common laboratory solvents, the

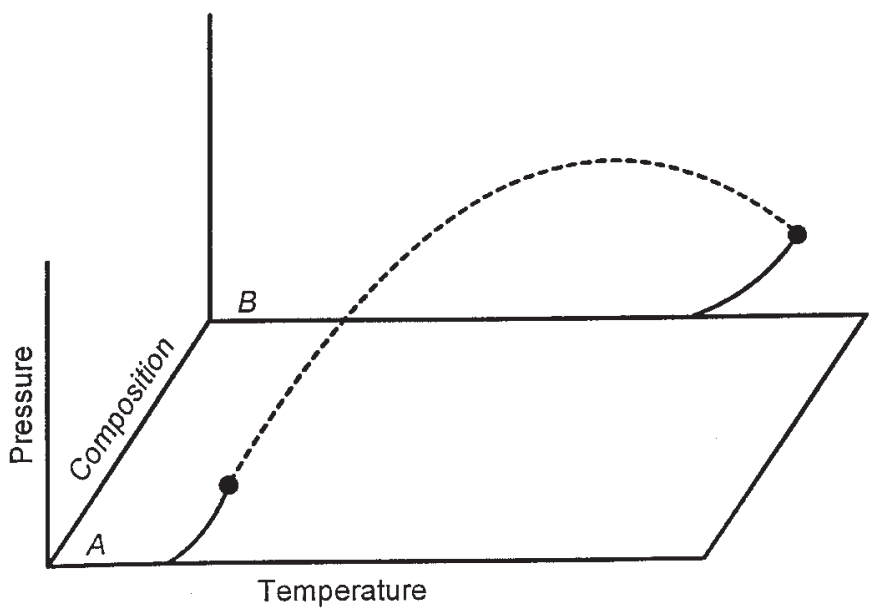

Figure 1. The critical locus for a Type-I binary mixture of components $A$ and $B$ spans the complete composition range of the mixture. The boiling lines for pure $A$ and for pure $B$ are shown as solid lines in their respective pressure-temperature planes at the limits of the composition axis. Each boiling line ends at its critical point.

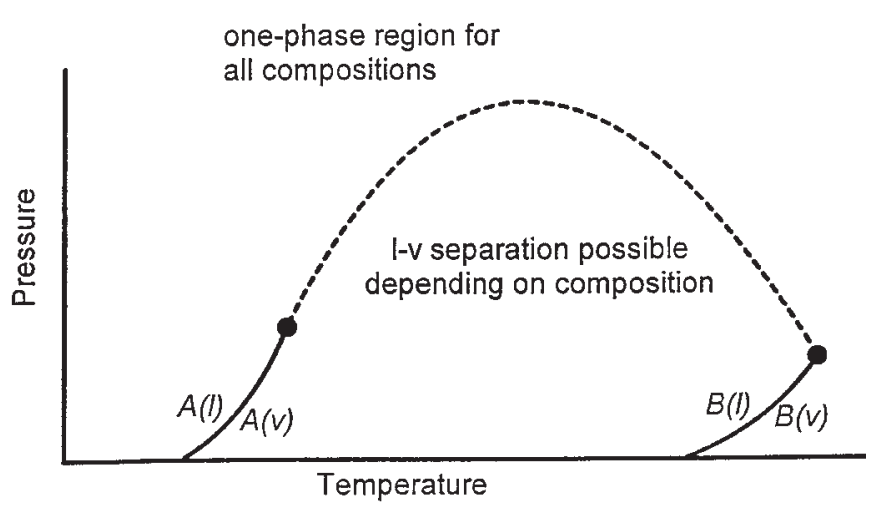

Figure 2. This is a projection of figure 1 along the composition axis showing the boiling lines for pure $A$ and $B$ and the critical locus as before. Only one fluid phase is possible in the outer region. Note that this region connects the liquid form of $\boldsymbol{A}$ with the vapor form of $B$ without a phase transition. Liquid-vapor separation is possible in the inner region, depending on the composition of the mixture.

notable exception being water. Figure 3 shows the critical loci for 14 solvent- $\mathrm{CO}_{2}$ mixtures [6,7]. The curve for 1 -octanol is not connected to the critical point for $\mathrm{CO}_{2}$ because the mixture is likely Type III $[8,9]$. However, its behavior is identical with Type I over the temperature range of the measured data. In addition, anyone with SFC equipment can quickly generate the pressure-temperature points of the critical locus for solvent mixtures not listed using a 
simple, flow-injection procedure which detects phase separation by its effect on the solvent peak shape $[6,7]$.

\section{Peak focusing mechanisms}

If open-tubular SFC were performed at optimal mobile phase velocity on 50- $\mu \mathrm{m}$-ID columns of a few meters length, it would be necessary to have initial solute bands in space of no more than a few millimeters to avoid contributing significantly to the broadening of peaks observed at the column outlet. Initial bands of about $10 \mathrm{~mm}$ or more are tolerable when higher velocities or longer columns (10 m or more) are used. However, an injection of only $100 \mathrm{nl}$ would require almost a 51-mm length of column just to contain the volume of the injected plug. If a separate liquid phase can exist at the temperature and pressure in use, the action of the mobile phase on a liquid plug will spread it into a flooded zone several meters in length [6,10-14]. Solutes will, of course, be distributed over this entire flooded zone by the liquid solvent. If, at the initial temperature and pressure, a separate liquid phase is not formed, then the injection solvent will be totally dissolved in the mobile phase, thus strengthening it tremendously. The result of making such an injection is, of course, large initial peak widths of poor shape, and premature peak elution due to transport of the solutes well past the column inlet by the sample solvent. However, initial peaks several meters in length can easily be focused in narrow plugs at the column inlet.

Focusing based on a spatial discontinuity in peak velocity is the easiest means of accomplishing this spatial compression. This is illustrated in figure 4 in which two tubes providing different retention factors for a solute are coupled. The velocity of a peak $p$ in tube $a$ is

$$
u_{p, a}=u_{m, a} /\left(1+k_{p, a}\right)
$$

where $u_{m, a}$ is the mobile phase velocity and $k_{p, a}$ is the retention factor for the peak while in tube $a$. An analogous relation exists in tube $b$. When a solute peak passes from tube $a$ to tube $b$, the spatial peak width is changed:

$$
\sigma_{b}=\sigma_{a} \frac{u_{m, b}\left(1+k_{p, a}\right)}{u_{m, a}\left(1+k_{p, b}\right)}
$$

where $\sigma_{b}$ and $\sigma_{a}$ are the spatial peak widths of the peak at the inlet of tube $b$ and at the outlet of tube $a$, respectively. Of course, since longitudinal transport is required to move the peak from tube $a$ to tube $b$, a very small amount of additional broadening, neglected in this explanation, will also be incurred. Therefore, if tube $b$ retains the peak more strongly than tube $a$, the peak will be shortened in space upon crossing the coupling if the mobile phase velocities are similar in both tubes. This change in spatial peak width occurs whenever two tubes providing different peak velocities are coupled. The difference in peak velocities may result even when tubes with the same stationary phase but different phase

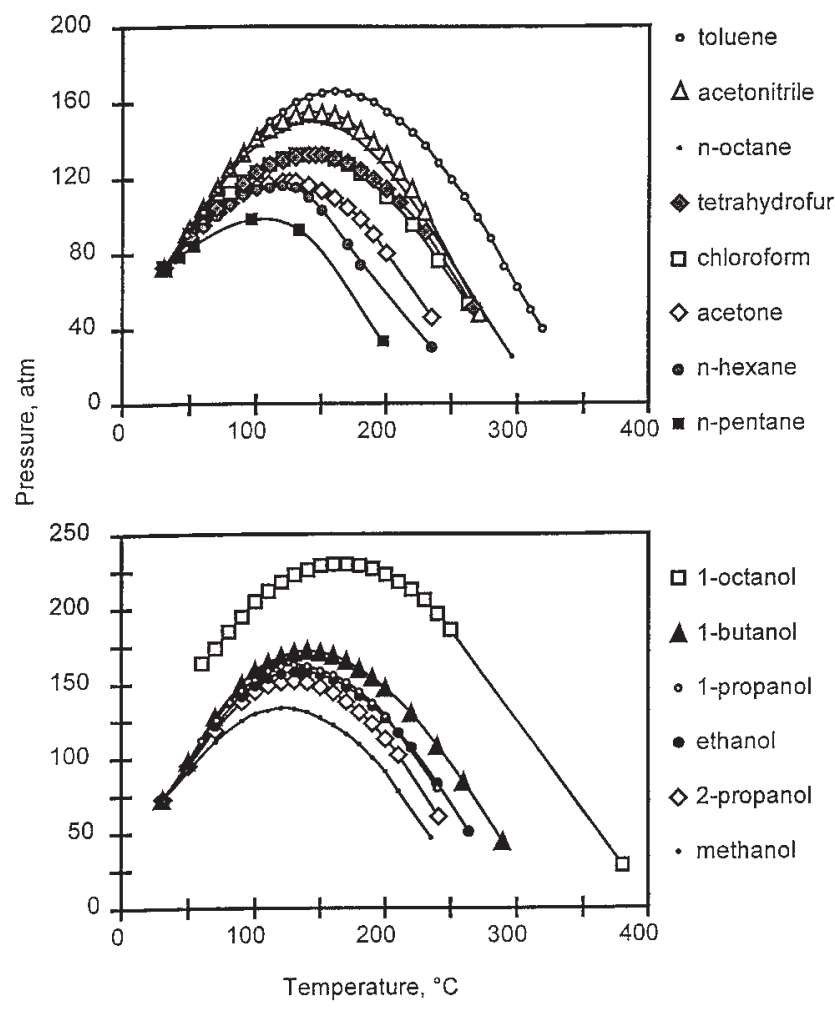

Figure 3. Experimental critical loci for 14 solvents in mixture with $\mathrm{CO}_{2}[6,7]$.
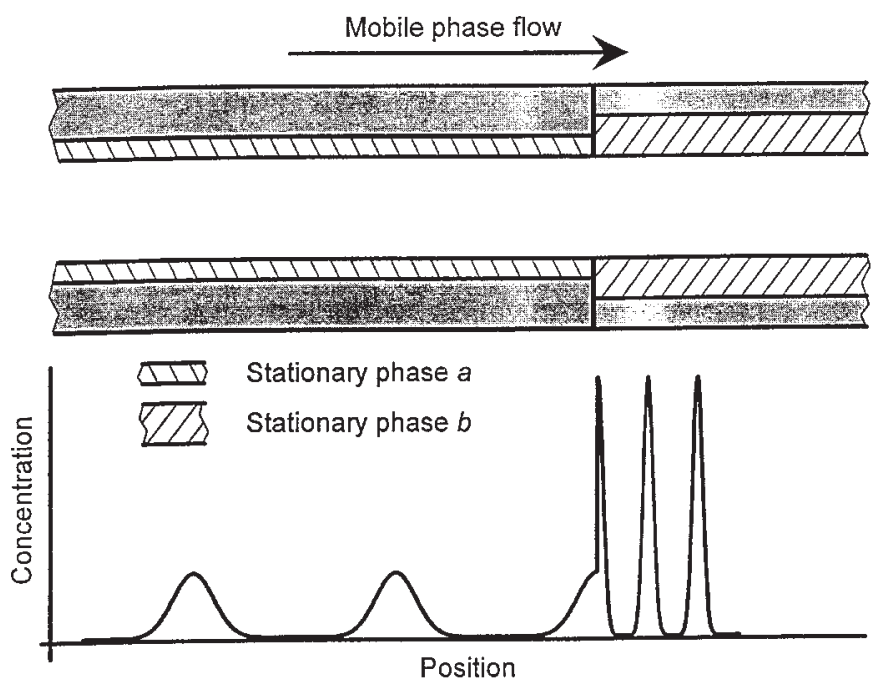

Figure 4. Illustration of phase ratio focusing of a peak. The individual peaks shown represent the position of a single solute peak at five evenly spaced time intervals. In this example the inner diameters of two joined columns are equal, but the stationary phase is more retentive in the second column, $b$, than in the first, $a$. The peak velocity on column $b$ is one-fourth the peak velocity on column $a$. 
ratio are chosen, thus giving us the name phase-ratio focusing [6,14-16]. Focusing will also occur when two columns with different stationary phases are coupled if the second has a slower peak velocity than the first.

Phase-ratio focusing is often taken to extreme by using an $a$ tube with no stationary phase at all, that is, a retention gap [6,14-16]. Its purposes are to contain flooding that occurs from the injection, to initially retain the solutes, and to accomplish at least a crude separation of the injection solvent from the solutes. Focusing is then accomplished by programming the mobile phase strength (through pressure, temperature, or composition gradients) so that the solutes begin to migrate on the retention gap but are still strongly retained upon reaching the column. The retention gap must be long enough to completely accommodate the flooded zone and prevent liquid sample solvent from reaching the column. The initial mobile phase conditions must be relatively mild so that solutes are not transported past the column inlet until all the sample solvent is evaporated and removed from the retention gap. Likewise, the program rate during the focusing step must not be so fast that a solute peak begins migrating on the column before it is entirely collected from the retention gap and focused-long retention gaps require more time and slower programming during focusing than do short ones. Peak widths may be reduced by a factor of 1000 or more by this technique.

We have used open-tubular columns to illustrate phaseratio focusing. Its use has been highly developed and widely adopted in open-tubular GC and SFC. Although there really is no analog of the retention gap in widespread use with packed columns, the principles of phase-ratio focusing still apply. For example, in HPLC, a sample solvent may be chosen which is significantly weaker than the mobile phase to allow large injection volumes. Of course, it is necessary that the solutes not be significantly retained by the injector or the tubing connecting it to the column. However, if the solutes are strongly retained by the column in the presence of injection solvent, significant focusing will occur according to equation 2 (where $a$ is now the inlet tube and $b$ is the column). Solutes will not begin migrating until the mobile phase replaces the sample solvent at the column inlet. Similarly, the mobile phase can be made weak initially, and a composition gradient applied to start solute migration on the column after the focusing step is complete.

Solvent trapping or focusing (that is, the solvent effect) is also possible in SFC [6,11-13]. This may occur on any tube, uncoated or coated with stationary phase, by exactly the same mechanism occurring in GC. The effect may also occur on packed SFC columns, but has not been extensively studied or reported. On open tubes, the sample solvent is distributed as a liquid film over a flooded section of column (or retention gap) where this liquid film acts as a temporary stationary phase. For solvent trapping it is necessary for the solutes to be mobile under injection conditions in the absence of the liquid solvent film, but essentially immobile in its presence. Further, the peak velocities of the solutes in the absence of the liquid film must be faster than the "velocity" at which the liquid film disappears. The liquid is evaporated by the application of fresh mobile phase from upstream. Liquid from the flooded zone is evaporated by the mobile phase until it is saturated with vapor. Thus, the liquid film evaporates from the inlet end of the flooded zone. The velocity at which the flooded zone shortens and disappears is therefore controlled by the evaporation rate. If the solute velocity in the absence of the liquid film is faster than the velocity at which the liquid film disappears, the solutes will accumulate in the moving tail of the flooded zone. When the last liquid disappears the now-narrow band of solutes is released to migrate normally.

In developing injection conditions requiring peak focusing, it is usually best to plan for phase-ratio focusing. This mechanism works well for solutes strongly retained under initial conditions where solvent trapping, if used alone, would fail. Peaks that are not well retained during injection usually undergo solvent trapping automatically if a liquid film of injection solvent forms. But, in any event, solutes retained on a retention gap may be easily refocused with proper attention to the mass transfer mechanism and rates.

\section{Dynamic-split (open-split) injection}

Dynamic-split (open-split) injection was formerly the most widely used sample introduction method on capillary columns. The injection is usually performed at room temperature using a 60-200 $\mathrm{nl}$ sample loop in an internal loop LC injector. In the splitter, part of the sample enters the column while the larger part of the sample moves outside the column until it is vented through a restrictor (Fig. 5a). Care must be exercised to position the column properly in the splitter assembly. Considerable peak area variations, depending on column positions, have been reported [17]. Also, by changing the dimensions of the split assembly, peak area variations of up to two orders of magnitude were obtained [18].

As in gas chromatography there is no linear relationship between peak area and split ratio, and the split ratio changes with sample viscosity and mobile phase density. If injections are made at different densities, good quantitation is difficult to obtain.

Most split systems function better with a high split ratio, i.e. with a high proportion of the sample going to waste. A high linear velocity of the fluid passing at the outside of the column is expected to improve the homogeneity of the sample. A high linear flow in the restrictor will also reduce the probability for accumulation of precipitated solutes in the restrictor, which otherwise leads to variations in the split ratio.

Based on raw peak areas, relative standard deviations of 2-8 \% have been obtained by open-split injection [18]. With internal standards this improved to $1 \%$ or better. With 

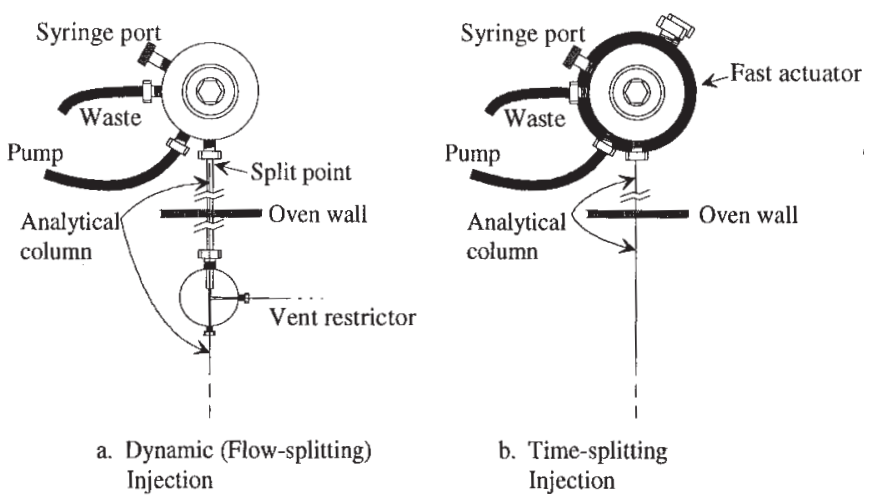

Figure 5. Sample introduction on capillary columns by dynamic splitting (a) and by time-splitting injection (b).

"difficult" solutes and certain restrictors lower performance can be expected.

\section{Time-splitting injection}

With precise computer timing in conjunction with highspeed pneumatics to move the injection valve from load to inject and back to load (Fig. 5b), the sample is split into one portion which is placed directly on the column and another portion which remains in the sample loop [19]. In the next loop-filling the remaining sample is removed. Less sample discrimination can be expected to be obtained with timedsplit injection, compared to open-split injection.

The relationship between peak area and injection time cannot be expected to be linear, and consequently non-linear time curves are usually found [20]. If the timed-split injector at the same time is equipped with a small open split, as on some instruments, the exact amount which is introduced on the column is not easily obtainable. The fraction of the sample which enters the column depends on the viscosity of the sample. With solvents of higher viscosity, smaller volumes are delivered to the mobile phase during the period in which the sample loop is connected to the mobile phase flow [21]. In general, quantitation by the internal standard method is recommended for this injection technique, as for all methods based on splitting.

Timed-split injection is usually considered to result in better reproducibility than open-split injection. With internal standards, less than $1 \%$ standard deviation was obtained by injection of n-alkanes [17], and similar results have been obtained with the injection of tocopherol [20]. With fatty acid methyl esters, approximately the same reproducibility was obtained with timed-split as with open-split injection [21].
A distinct advantage of timed-split injection is the removal of the solvent tail. The method also allows larger, more accurate, sample loops to be used with capillary columns.

\section{Solvent venting under supercritical conditions}

In the first solvent venting experiments on capillary SFC columns, sample volumes up to $0.5 \mu \mathrm{l}$ were injected on a 50- $\mu \mathrm{m}$-ID precolumn with complete solvent elimination [22]. With this technique, the solvent is more or less completely separated from the solutes on the precolumn by a partitioning mechanism, and the solutes are transferred to the main column and focussed at the column inlet. The major part of the solvent is vented through a restrictor at a rapid $(10-15 \mathrm{~cm} / \mathrm{s})$ but controlled flow rate. With a $2-\mathrm{m}$ precolumn the venting time was $15-20 \mathrm{~s}$. Discrimination losses occur for early eluting peaks if venting times are too long, while peak shapes are degraded when the starting pressures are too high [23]. Sample recovery can be quite acceptable with this technique. For example, Berg et al. [24] reported $100 \%$ recovery of diglycerides. They used a $7-\mathrm{m} \times 50-\mu \mathrm{m}-$ ID precolumn to introduce $1 \mu \mathrm{l}$ samples onto $50-\mu \mathrm{m}$-ID columns.

Based on absolute area measurements, the solvent venting technique resulted in better injection reproducibility $(\mathrm{RSD}=2-5 \%)$ compared to timed split injection $(\mathrm{RSD}=$ $6 \%$ ) and open-split injection $(\mathrm{RSD}=8 \%$ ) on the same equipment. Addition of an internal standard reduced the relative standard deviation to less than $1 \%$. With a minimum detectable quantity of 0.02-0.1 ng, sample concentrations of 0.1-0.5 ppm are within reach.

This method seems likely to be a method for introduction of sample volumes not larger than 1-2 $\mu$ l on 50- $\mu$ m-ID columns. The valve between the precolumn and the column produces some band broadening which must be reversed by peak focusing at the column inlet. The valve gives, however, additional opportunities such as using the system for coupled column separations.

\section{Solvent venting under reduced pressure}

Solvent venting by pressure reduction after mixing and dilution with supercritical $\mathrm{CO}_{2}$ [25], or by purging the sample with another gas [2], are techniques which also require a precolumn or a retention gap. By purging the sample into the precolumn with an inert gas, i.e. by GC conditions, the solvent is evaporated in the gas flow and carried rapidly through the column and out of the vent valve (Fig. 6). After the solvent is evaporated and the solutes are coated on the walls of the precolumn, the purging valve is switched back to the carbon dioxide line, and liquid or supercritical $\mathrm{CO}_{2}$ is 


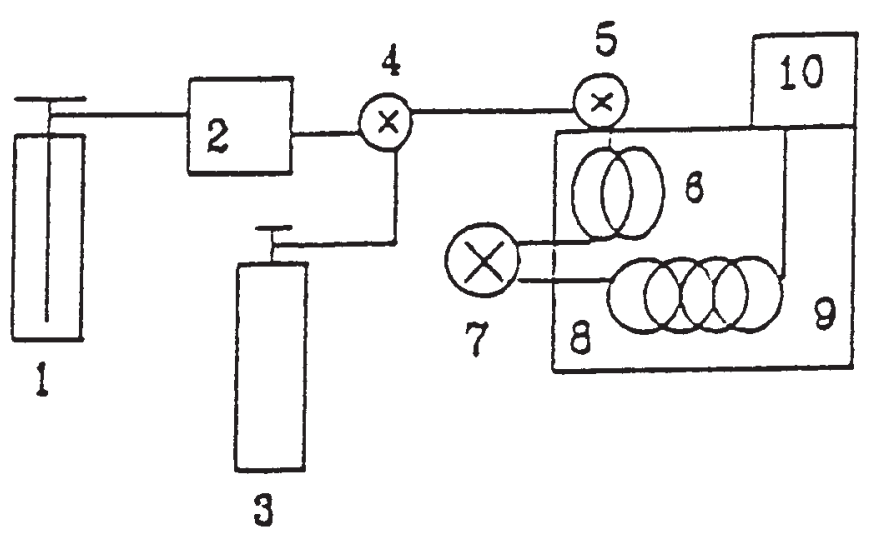

Figure 6. Solvent venting with gas purging: (1) $\mathrm{CO}_{2}$, (2) pump, (3) helium tank, (4) valve, (5) injector, (6) precolumn, (7) vent valve, (8) column, (9) oven, (10) detector.

introduced. The solutes which dissolve in the flow can be focused by a temperature gradient at the column entrance [2] or a temperature gradient on the precolumn [26]. Rapid expansion of carbon dioxide in a wide precolumn $(20 \mathrm{~cm} \times$ $200 \mu \mathrm{m}$ ID) and a temperature difference between the precolumn and the column added to the band focusing [26].

One advantage with gas purging injection is the opportunity to do multiple injections. For strongly retained solutes (n-alkanes with 32-38 carbon atoms) five multiple injections of $1 \mu \mathrm{l}$ resulted in complete recovery. The reproducibility of $0.5 \mu 1$ injections was RSD $=4-1 \%$, based on raw areas. Compared to split injection, the column efficiency was reduced by $15-20 \%$. By using longer, narrow precolumns with 11-15 $\mu \mathrm{m}$ ID, the efficiency improved to the level of split injection.

Another gas-purging method, with helium, made use of a $1.5-\mathrm{m} \times 100-\mu \mathrm{m}-\mathrm{ID}$ uncoated retention gap [24]. By injecting $1 \mu \mathrm{l}$ samples of glycerides, triglycerides were completely recovered, while diglycerides suffered a $30 \%$ loss. Even after 10-50 multiple injections of $1 \mu \mathrm{l}$ each, the triglycerides were completely recovered. Sample volumes of $1 \mu \mathrm{l}$ were injected with RSD $=2-7 \%$ without internal standards in manual mode, and less than $1 \%$ RSD in automated mode. The same technique, with a 1.8-m-long retention gap, was developed into a fully automated injector with a robotic autosampler (Fig. 7) for determination of polymer additives from food simulated liquids [27].

With a combination of sample dilution and solvent venting, up to $100-\mu 1$ samples of triglycerides were injected on a $50-\mu \mathrm{m}-\mathrm{ID}$ precolumn in combination with a $100-\mu \mathrm{m}-\mathrm{ID}$ column [25]. Concentrations as low as a few ppb could be analyzed, when solvents of very high purity were utilized.

Packed precolumns can also be used for solvent elimination. A $6-\mathrm{cm} \times 200-\mu \mathrm{m}-$ ID capillary, filled with polyoctylsiloxane coated particles, was connected to a $50-\mu \mathrm{m}-\mathrm{ID}$

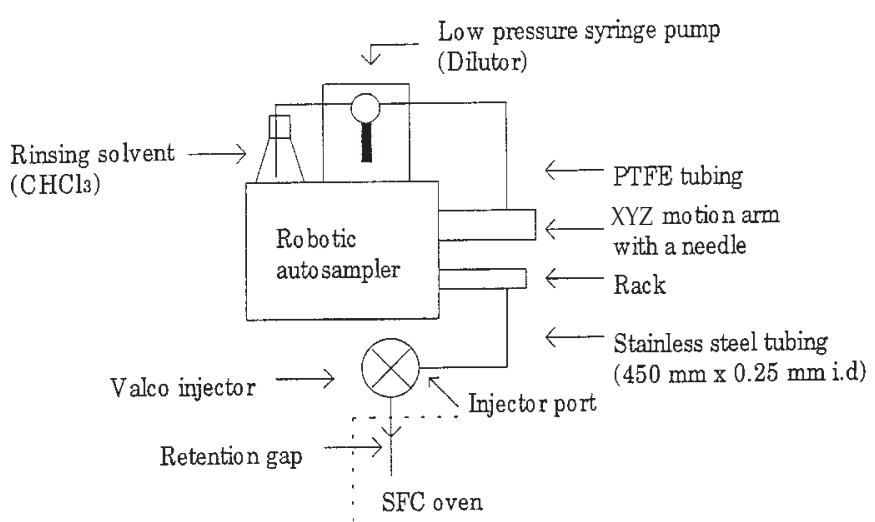

Figure 7. Robotic autosampler coupled to SFC with solvent venting.

column. With a valve which kept high pressure on the main column, with gas purging application and backflush elution from the packed precolumn, the column efficiency was essentially equal to the efficiency obtained by split injection [3].

Another sophisticated method, combining solvent venting with pressure focusing, which was developed by Campbell et al. [28] allows large volumes (100 $\mu$ l or more) to be injected. Although excellent results have been reported, the equipment is considered too complicated for use by many laboratories. It is also expensive, not commercially available, and is patented.

\section{Direct injection using a retention gap}

This technique [6,29] effectively utilizes phase behavior of the sample solvent-mobile phase mixture combined with peak focusing to allow direct injections of up to about $1 \mu \mathrm{l}$ volume onto $50-\mu \mathrm{m}$-ID open-tubular SFC columns. The experimental setup is shown in figure 8 .

The injection is performed at room temperature, outside the column oven, where $\mathrm{CO}_{2}$ is liquid, not supercritical fluid. The sample solvent is chosen from among those miscible with the $\mathrm{CO}_{2}$ mobile phase as liquids so that no phase separation occurs in the valve and the transfer tubing. The purpose of avoiding phase separation is to ensure good transfer efficiency of sample to the oven. However, the initial pressure and oven temperature are chosen so that the $\mathrm{CO}_{2}$-solvent mixture will separate into liquid and vapor phases when the sample plug reaches the oven; that is, the pressure and oven temperature must be set so that when a plug of solvent (containing dissolved sample components) enters the oven in a stream of mobile phase (that is, increases temperature at constant pressure), the plug will cross the critical locus 


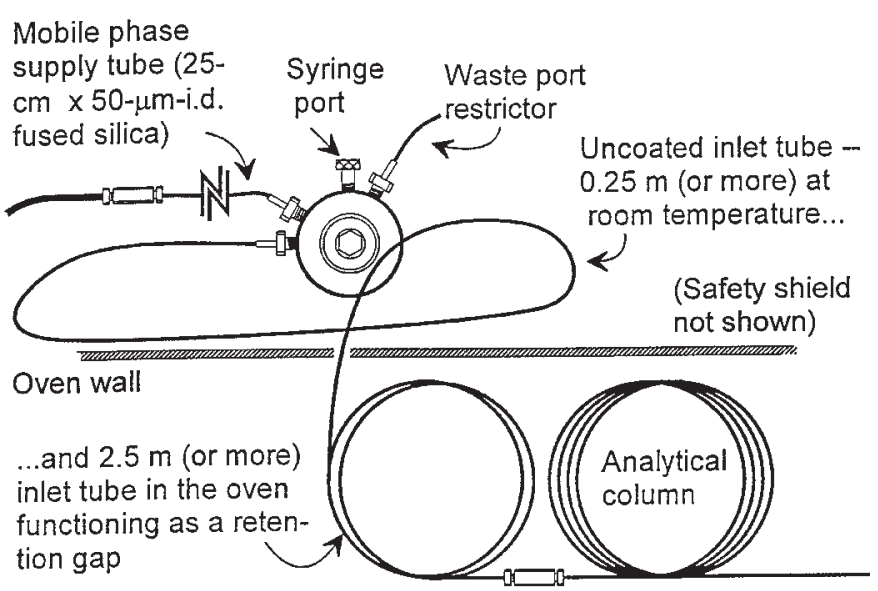

Figure 8. Experimental setup for direct injection onto a retention gap.

(Fig. 2). Thus, knowledge of the critical loci for mixtures of $\mathrm{CO}_{2}$ and common solvents (Fig. 3) are necessary for setting the initial parameters. All this is necessary to prevent solutes and liquid injection solvent from reaching the column inlet before separating the solutes from the injection solvent. The liquid is evaporated and removed as vapor by the mobile phase, but solutes are either trapped in the remaining liquid, or simply remain on the retention gap at this step. After the solvent is removed, the solutes are transported from the retention gap to the column inlet and focused there by

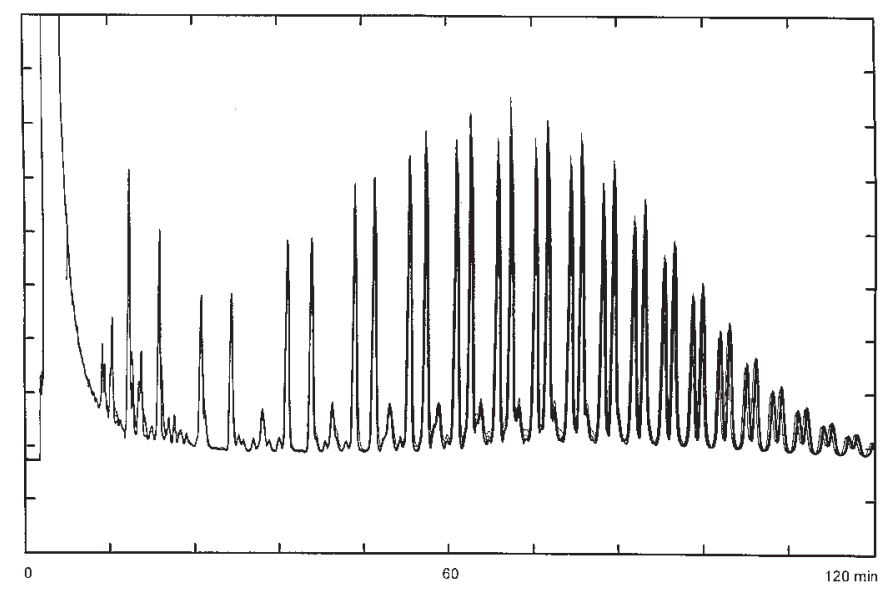

Figure 9. Ten superimposed chromatograms of Neodol 23-6.5 (a surfactant made by ethoxylation of $n-C_{12}$ and $n-C_{13}$ alcohols) dissolved in toluene and injected directly onto a retention gap. Other conditions: $0.06-\mu \mathrm{l}$ injections; $2-\mathrm{m}$ x 50- $\mu \mathrm{m}$-ID retention gap, with the first $0.25 \mathrm{~m}$ at room temperature; $10-\mathrm{m} \times 50-\mu \mathrm{m}-$ ID biphenyl column (Dionex, Salt Lake City, UT); oven temperature is $120^{\circ} \mathrm{C}$; pressure program is $7-8 \mathrm{MPa}$ at $0.1 \mathrm{MPa} / \mathrm{min}$, then 8-41.5 $\mathrm{MPa}$ at $0.3 \mathrm{MPa} / \mathrm{min}$ [34]. phase-ratio focusing as described earlier. This is usually accomplished using an isothermal pressure or density program.

There are several advantages of this technique. It is very inexpensive and instrumentally simple, requiring only the addition of the retention gap and one union between the injector and the column in a system that is otherwise minimal. No unusual operator skill is required since, once sufficient parameters are selected, the individual details of the mass transfer process (transfer, liquid flooding, solvent evaporation, and solute focusing) all occur spontaneously. The injector is left in the inject position long enough to ensure an essentially quantitative transfer of the solutes into the system. The injector is then returned to the load position to improve the shape of the trailing edge of the solvent peak. However, any potential tailing of the solute peaks due to injection is eliminated during the focusing step. Chromatograms are highly reproducible as illustrated in figure 9, with peak-area and retention-time relative standard deviations around $1 \%$. Injections of $0.5 \mu \mathrm{l}$ can easily be accomplished, thus providing part-per-million detection limits with many ordinary detectors.

\section{Injection on packed columns}

Direct injection is the standard method of sample introduction in packed-column SFC. With regular size packed HPLC columns, injection in SFC is a fairly simple technique. Injection volumes of $20 \mu \mathrm{l}$ can be accommodated on 4.6mm-ID columns, without peak focusing [30]. The nature of the sample solvent may affect peak shapes, as in HPLC, but the impact of sample solvents on packed SFC columns has not been examined as thoroughly as injection on open-tubular columns. With modified mobile phases, solvent effects from the sample solvents are usually of less importance. Also, aqueous samples can be tolerated, at least in $\mathrm{CO}_{2}$ modified with methanol [30].

Solvent elimination has mainly been used with opentubular capillary columns, but Oudsema and Poole [31] have studied similar techniques for packed microbore (1 mm ID) columns. By gas purging (helium) the sample into a steel retention gap, 1-100 $\mu$ l volumes could be injected with good reproducibility.

With preparative-size columns, Crétier et al. used heated helium to remove the solvent on a precolumn [32]. Preparative separations require concentrated sample applications, with more emphasis on solubility than on elution strength in choosing sample solvents [33].

\section{Introduction of supercritical fluid extracts}

The method for transferring an aliquot of the extract or the complete extract depends on the extraction procedure. 
In dynamic extractions the fluid is pumped continously through the extractor to the collector. If a static extraction is performed, an aliquot is sampled by a valve at intervals. In analytical procedures, dynamic extraction requires the presence of a sample-concentrating unit, based on cooling (cold trap), heating (density focusing) in combination with a precolumn, or solute precipitation in a piece of tubing by venting towards the atmosphere. Depending mostly on the volatility of the sample constituents, the solute collection will be more or less quantitative.

An advantage of using the mobile phase for extraction is that the whole extract can be expected to elute. As for other chromatographic systems, there is never a guarantee that all the constituents of a sample dissolved in a solvent will be eluted, depending on the solubility in the mobile phase. However, one should not forget that if the extraction has been performed at high density, elution of all components can be expected to require a density gradient with a high density end. The only possibility of losing part of the sample then is by using columns with higher adsorptive properties than the original sample matrix.

\section{Sample losses in the valves}

With pressures possibly approaching $50 \mathrm{MPa}$, occasional leaks are bound to take place in a rotating valve. Many valves are guaranteed leak-proof to $40 \mathrm{MPa}$, but even below this limit leaks occur. Leaks are often a result of the impact of solid particles between the rotor and the stator, particles coming from the sample, the fluid container, or from the end of the connecting tubing. Particle filters should be inserted in the line prior to the injector. Fused silica tubing, both at the inlet and the outlet, should be connected with care to avoid splintered ends. Samples with suspended particles must be filtered or centrifuged.

Leaks are not easily detected in capillary GC or LC since the fluid flow which usually escapes is very low. Leaks are easier to find in SFC since the mobile phase expands 3001000 times when vented to atmosphere through a leak. Leaks will not affect retention if pressure-controlled pumps are used. A close inspection of the stator and the rotor usually will reveal whether there is a leak in the injector. As long as the injector is not leaking at the lower pressure during injection, the peak height will not be affected, even if a leak occurs at higher pressure.

By injecting the sample dissolved in a volatile solvent in a hot injector, part of the sample will be lost. Even when the injector is not intentionly heated, waste oven heat may warm the valve to a temperature where the expanding gas from evaporating diluent can expel part of the liquid sample, depending on the valve port assignment. A waste-port restrictor or a valve cooling system can eliminate such problems [29].
If the yield transferred to the column is different for different solutes, the term discrimination is commonly used. Discrimination may take place when a part of the sample is split away, as with open-split injection, solvent elimination and similar methods. Volatile or rapidly eluted components are transferred to the column to a smaller extent than less volatile (more retained) components. Claims of no discrimination with split systems should always be regarded as sample-related statements, and the actual extent of discrimination in every case needs to be determined with reference solutions. For the majority of applications in SFC, however, discrimination effects are not of major impact.

\section{References}

1. Scott, R. P. W.; Simpson, C. F. J. Chromatogr. Sci. 1982, 20, 62.

2. Lee, M. L.; Xu, B.; Huang, E. C.; Djordjevic, N. M.; Chang, H.-C. K.; Markides, K. E. J. Microcol. Sep. 1989, $1,7$.

3. Koskii, I. J.; Markides, K. E.; Richter, B. E.; Lee, M. L. Anal Chem. 1992, 64, 1669.

4. Tuominen, J. P.; Markides, K. E.; Lee, M. L. J. Microcol. Sep. 1991, 3, 229.

5. Van Konynenburg, P. H.; Scott, R. L. Phil. Trans. R. Soc. of London, A. 1980, 298, 495.

6. Chester, T. L.; Innis, D. P. J. Microcol. Sep. 1993, 5, 261-273.

7. Ziegler, J. W.; Dorsey, J. G.; Chester, T. L.; Innis, D. P. Anal. Chem. 1995, 67, 456.

8. Ziegler, J. W.; Chester, T. L.; Innis, D. P.; Page, S. H.; Dorsey, J. G. In Innovations in Supercritical Fluids: Science and Technology; (Hutchenson, K. W.; Foster, N. R. Eds); ACS Symposium Series No. 608; American Chemical Society: Washington, DC, 1995; Chapter 6.

9. Ziegler, J. W.; Ph.D. Dissertation, University of Cincinnati, 1996.

10. Fairbrother, F.; Stubbs, A. E. J. Chem. Soc. 1935, 1, 527.

11. Grob, K.; Grob Jr, K. J. High Resolut. Chromatogr. \& Chromatogr. Comm. 1978, 1, 57.

12. Grob Jr, K. J. Chromatogr. 1983, 279, 225.

13. Grob, K.; Schilling, B. J. Chromatogr. 1983, 264, 7.

14. Grob, K. J. Chromatogr. 1982, 237, 15.

15. Grob, K.; Muller, R. J. Chromatogr. 1982, 244, 185.

16. Grob, K.; Karrer, G.; Riekkola, M.-L. J. Chromatogr. 1985, 334, 129.

17. Richter, B.; Knowles, D. E.; Andersen; M. R.; Porter, N. L.; Campbell, E. R.; Later, D. W. J. High Resolut. Chromatogr. 1988, 11, 29.

18. Köhler, J.; Rose, A.; Schomburg, G. J. High Resolut. Chromatogr. 1988, 11, 191.

19. Harvey, M. C.; Stearns, S. D. J. Chromatogr. Sci. 1983, 21, 473.

20. Greibrokk, T.; Berg, B. E.; Johansen, H. In International Symposium on Supercritical Fluids, Nice, Perrut, M., Ed.; October 1988, 425.

21. Schomburg, G.; Behlau, H.; Haüsig, U.; Hoening, B.; Roeder, W. J. High Resolut. Chromatogr. 1989, 12, 142.

22. Farbrot Buskhe, A.; Berg, B. E.; Gyllenhaal, O.; Greibrokk, T. J. High Resolut. Chromatogr. 1988, 11, 16. 
23. Berg, B. E.; Greibrokk, T. J. High Resolut. Chromatogr. 1989, $12,322$.

24. Berg, B. E.; Flaaten, A.M.; Paus, J.; Greibokk, T. J. Microcol. Sep. 1991, 3, 17.

25. Hirata, Y.; Kadota, Y.; Konodo, T. J. Microcol. Sep. 1991, 3, 17.

26. Liu, Z.; Farnsworth, P. B.; Lee, M. L. J. Microcol. Sep. 1992, 4, 199.

27. Berg, B. E.; Greibrokk, T. J. High Resolut. Chromatogr. 1994, 17, 49.

28. Campbell, R. M.; Cortes, H. J.; Green, L. S. Anal. Chem. 1992, 64, 2852.
29. Chester, T. L.; Innis, D. P. Anal. Chem. 1995, 67, 3057.

30. Berger, T. A. Chromatographia 1995, 41, 471.

31. Oudsema, J. W.; Poole, C. F. J. High Resolut. Chromatogr. 1992, 15,65 .

32. Cretier, G.; Majdalani, R.; Rocca, J. L. Chromatographia 1990, 30, 645 .

33. Coleman, K.; Boutant, R.; Vérillon, F. Isolation and Purification 1999, 3, 9.

34. Chester, T. L.; Innis, D. P. The 7th International Symposium on Supercritical Fluid Chromatography and Extraction, Indianapolis, IN, March 31-April 4, 1996. 